\title{
Motor fitness and preschooler children obesity status
}

\section{Sandra Silva-Santos, Amanda Santos, Susana Vale \& Jorge Mota}

To cite this article: Sandra Silva-Santos, Amanda Santos, Susana Vale \& Jorge Mota (2016): Motor fitness and preschooler children obesity status, Journal of Sports Sciences, DOI: 10.1080/02640414.2016.1232486

To link to this article: http://dx.doi.org/10.1080/02640414.2016.1232486

册 Published online: 15 Oct 2016.

Submit your article to this journal \lceil

Џ Article views: 80

View Crossmark data \lceil 


\title{
Motor fitness and preschooler children obesity status
}

\author{
Sandra Silva-Santos ${ }^{\mathrm{a}}$, Amanda Santos ${ }^{\mathrm{a}}$, Susana Vale ${ }^{\mathrm{a}}$ and Jorge Mota (i) $^{\mathrm{a}}$

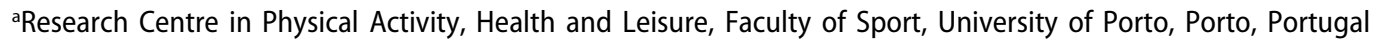

ABSTRACT

The aim of this study was to examine the association between motor fitness (MF) and obesity status in preschool children.

The sample comprised 467 children aged 3-6 years. Preschool children body mass index was classified according to International Obesity Task Force and categorised into three levels, normal, overweight and obesity. Total physical activity was assessed by accelerometer and MF test was assessed through two MF tests $10 \times 5 \mathrm{~m}$ shuttle run test (SRT) and a $7 \mathrm{~m}$ jumping distance on 2 feet test (J2F). Low MF was considered for MF if SD above 1. A single variable with three categories was created: low MF medium MF and high MF.

The prevalence of normal weight, overweight and obesity was $67.6 \%, 22.7 \%$ and $9.7 \%$, respectively. The prevalence of SD > 1 for SRT was $13.7 \%$ and $14.4 \%$ for J2F, for single variable was $19.2 \%$. Multinomial logistic regression analysis showed that obese preschoolers were more likely six times classified as having low MF level than their non-overweight counterparts (OR: 6.4; IC: 1.3-36.6).

This study showed a considerable prevalence of overweight and obesity among preschoolers. Obesity has already been associated with lower MF. Further longitudinal studies are needed to confirm this data.
ARTICLE HISTORY

Accepted 23 August 2016

KEYWORDS

Physical fitness; motor fitness; preschool children; body mass index

\section{Introduction}

The high prevalence of childhood overweight and obesity have been cause for great concern since detrimental biological and psychological consequences of childhood overweight have been described. In addition, it was recognised that fitness and physical activity (PA) are associated with obesity. For instance, children and adolescents with overweight are less fit than their leaner peers (Kriemler et al., 2008; Ortega, Ruiz, \& Castillo, 2013), while there is growing evidence that fitness during childhood and adolescence is negatively associated with total and central body fat in cross-sectional studies as well as later in life (Cadenas-Sanchez, Artero, Concha, Leyton, \& Kain, 2015; Ortega et al., 2007).

Whereas physical fitness is considered a powerful health marker in children and adolescents (Ortega, Ruiz, Castillo, \& Sjöström, 2008; Smith et al., 2014), motor fitness (MF) is a component of physical fitness that is related with the improvement of performance in sports and motor skills (Ortega et al., 2015). The main components of MF are speed, agility and balance (Caspersen, Powell, \& Christenson, 1985; Ortega et al., 2015). Secular trends have shown a decrease for most of the basic motor abilities (Klein, Manz, Ferrari, Struder, \& Graf, 2015). Although the preschool years are a critical period in terms of developing these components, available data are scarce (Klein et al., 2015). Therefore, in this age, it is very important to direct special attention in satisfying and developing fundamental motor skills, which enable children to overcome space, obstacles and resistance and to manipulate objects. Additionally, improvements in MF have shown to reduce the risk of becoming overweight/obese in adolescence (Haga, 2009; Moliner-Urdiales et al., 2011). However, the data addressing the relationship of MF with obesity status in preschool children is scarce (Bayer, Bolte, Morlock, Rückinger, \& von Kries, 2009). Thus, the aim of this study was to examine the association between the MF and the obesity status in preschool children.

\section{Methods}

\section{Participants and data collection}

This was a cross-sectional study completed in kindergartens enrolled in the Preschool PA, Body Composition and Lifestyle Study (PRESTYLE), a longitudinal study that began in the autumn of 2012. All kindergartens located in the metropolitan area of Porto were invited to participate and 20 classrooms were selected. All children belonging to the selected classrooms were invited to participate. A random sample of 1160 children, aged 3-6 years, was recruited from kindergartens located in the metropolitan area of Porto, Portugal. Only 467 preschoolers met the inclusion criteria (52.8\% girls). In this study, we have included results in the shuttle run test (SRT), $7 \mathrm{~m}$ jumping distance on 2 feet test (J2F) and total physical activity time (TPA). Height and weight information has also been included. Data collection took place between April 2013 and November 2014. A written consent was obtained from parents and school supervisors. Study procedures were approved by the Portuguese Foundation for Science and Technology and by the Scientific Board of Physical Activity and Health PhD programme.

CONTACT Sandra Silva-Santos sandrcris@gmail.com Rua Dr. Plácido Costa n.91, 4200-450, Porto, Portugal

Present affiliation for Susana Vale is Research in Department of Sport Sciences, High School of Education, Polytechnic Institute of Porto, Porto, Portugal

๑) 2016 Informa UK Limited, trading as Taylor \& Francis Group 


\section{Anthropometric measures}

Body mass and height were measured by standard anthropometric methods. Body mass was measured to the nearest $0.10 \mathrm{~kg}$, with participants lightly dressed (underwear and t-shirt only) using a portable digital scale (Tanita Inner Scan BC 532, Tokyo, Japan). Height was measured to the nearest millimetre in bare or stocking feet with children standing upright against a Holtain portable stadiometer (Tanita). The measurements were repeated twice and the average was recorded. Body mass index was calculated and classified according to International Obesity Task Force into three groups, normal weight, overweight and obesity (Cole, Bellizzi, Flegal, \& Dietz, 2000).

\section{MF}

For assessing MF we used the two MF tests, $10 \times 5 \mathrm{~m} \mathrm{SRT} \mathrm{and}$ jumping a distance of $7 \mathrm{~m}$ on 2 feet (J2F). Some studies suggested that SRT and J2F are reliable tests to be used for assessing MF (speed/agility) in preschool children (Fjortoft, Pedersen, Sigmundsson, \& Vereijken, 2011; Haga, 2008). Each MF test was standardised and standard deviation above one (SD > 1) was considered low motor abilities. Afterwards, MF was categorised as low MF and high MF. Afterwards, both MF tests were associated and a single variable was created with three categories: low MF (SD > 1 for SRT and J2F); medium MF (SD > 1 for SRT or J2F) and high MF (SD $<1$ for SRT and J2F). Although several studies have examined the construct validation of MF tests, the majority assessed the overall scores of the subset rather than the single test measurements (Fjortoft et al., 2011; Fjørtoft, Pedersen, Sigmundsson, \& Vereijken, 2003). Therefore, little information is available about which test is the most valid in this age group. In addition, no criterion-related validity or health-related studies (either longitudinal or cross-sectional) were identified (Fjortoft et al., 2011).

Children were tested individually. The SRT and JSF were explained and demonstrated before the child started. If a child made an error, instructions and demonstrations were repeated, and the child was allowed a new attempt. If a second error occurred or if a child could not perform the test, then the MF test was scored as missing.

\section{PA}

Daily PA was measured using the ActiGraph GT1M accelerometer (Pensacola, FL, USA). This accelerometer provides output in activity counts, which gives information about the intensity of PA (Janz, 1994). Alternatively, accelerometer output can be interpreted using specific cut-points, which identify time in different intensities of PA. Data reduction, cleaning and analyses of accelerometer data were performed using a specially written program described and used previously (Purslow, Hill, Saxton, Corder, \& Wardle, 2008; Sardinha, Baptista, \& Ekelund, 2008). Data were analysed using specific paediatric cut-points, which have been validated for young children: $\geq 150 \mathrm{cpm}$ for active time (total PA time (TPA) as already been used in several studies (Byun, Beets, \& Pate, 2015; Byun, Dowda, \& Pate, 2011; O'Connor et al., 2014; Pate et al., 2015). For the purpose of this study, the epoch duration or sampling period was set to $5 \mathrm{~s}$, which is more accurate for the spontaneous and intermittent activities of children as used previously with a similar sample (Vale, Santos, Silva, SoaresMiranda, \& Mota, 2009). PA was monitored for seven consecutive days (Monday to Sunday). A minimum of $10 \mathrm{~h}$ of data per day was required for analysis. Periods of $60 \mathrm{~min}$ or more of continuous zeroes were considered non-wear times and were excluded from the analyses. Parents were instructed to attach the accelerometer when the child awoke and to remove it when they went to bed. The accelerometer was worn snuggly under clothing on the child's hip using a fully adjustable elastic waist belt.

\section{Statistical analyses}

Means and SD were calculated to describe children's characteristics by sex. It was analysed one association between each MF test and weight status by binary logistic regression.

The association between weight status and single variable (SRT+J2F) was examined using multinomial logistic regression. The model was adjusted by age and TPA. Statistical analysis was performed using the SPSS 21.0 software (SPSS Inc., Chicago, IL, USA). The level of significance was set at alpha level of 0.05 .

\section{Results}

Table 1 shows descriptive characteristics of the sample according to gender, boys were significantly more active than girls $(P=0.000)$. Among girls, the prevalence of overweight and obesity was $23.8 \%$ and $12.6 \%$, respectively. In comparison, the prevalence of overweight and obesity among boys was lower at $21.6 \%$ and $6.3 \%$, respectively; this difference was statistical significance $(P=0.014)$. No other statistical significant differences were found.

Our sample has shown that $84.0 \%$ normal weight children had high MF, $14.9 \%$ medium and $1.1 \%$ low. Children with overweight, $81.3 \%$ had high $\mathrm{MF}, 17.7 \%$ medium and $1.0 \%$ low. Finally, $71.1 \%$ obese children had high MF, $23.7 \%$ medium and $5.3 \%$ high.

Binary logistic regression analysis (Table 2) showed that obese preschoolers were 4 times more likely to be classified as having low MF level in SRT than their non-overweight

Table 1. Preschool children's anthropometric and demographic characteristics.

\begin{tabular}{lcccc}
\hline & \multicolumn{1}{c}{ Total } & Girls & \multicolumn{1}{c}{ Boys } & \\
\cline { 2 - 4 } Variables & $(n=467)$ & $(n=244)$ & $(n=223)$ & $P$-value \\
\hline Weight $(\mathrm{kg})$ & $20.4 \pm 3.8$ & $20.6 \pm 4.1$ & $20.1 \pm 3.5$ & 0.202 \\
Height $(\mathrm{cm})$ & $109.0 \pm 6.4$ & $109.1 \pm 6.6$ & $109.0 \pm 6.2$ & 0.854 \\
BMI (kg/m $\left.{ }^{2}\right)$ & $17.0 \pm 1.9$ & $17.1 \pm 2.1$ & $16.8 \pm 1.7$ & 0.098 \\
TPA (min per week) & $20.3 \pm 5.5$ & $265.3 \pm 49.7$ & $289.0 \pm 51.5$ & 0.000 \\
J2F (s) & $5.7 \pm 1.5$ & $5.7 \pm 1.4$ & $5.8 \pm 1.7$ & 0.526 \\
SRT (s) & $27.0 \pm 2.9$ & $27.1 \pm 2.7$ & $26.9 \pm 3.1$ & 0.577 \\
& $\%$ & $\%$ & $\%$ & \\
BMI group & & & & \\
Normal weight $\left(\mathrm{kg} / \mathrm{m}^{2}\right)$ & 67.6 & 63.5 & 72.1 & 0.014 \\
Overweight $\left(\mathrm{kg} / \mathrm{m}^{2}\right)$ & 22.7 & 23.8 & 21.6 & \\
Obese $\left(\mathrm{kg} / \mathrm{m}^{2}\right.$ & 9.7 & 12.7 & 6.3 & \\
\hline
\end{tabular}

BMI, body mass index; $\geq 3 \mathrm{~h}$ TPA, at least $3 \mathrm{~h}$ total physical activity per day each day a week; J2F, jumping a distance of $7 \mathrm{~m}$ on 2 feet; SRT, $10 \times 5 \mathrm{~m}$ shuttle run test; bold figures indicate significant $P$-values. 
Table 2. Association between performance in shuttle run test (SRT) and jumping 2 feet test (JSF) and obesity status in preschoolers.

\begin{tabular}{llllllll}
\hline & \multicolumn{3}{c}{ SRT } & & \multicolumn{3}{c}{ J2F } \\
\cline { 2 - 4 } \cline { 6 - 8 } BMI tests & OR $(95 \% \mathrm{Cl})$ & $R^{2}{ }_{\mathrm{N}}$ & $P$-value & & $\mathrm{OR}(95 \% \mathrm{Cl})$ & $R^{2}{ }_{\mathrm{N}}$ & $P$-value \\
\hline Normal weight & 1 & 0.27 & 1 & & 1 & 0.28 & 1 \\
Overweight & $0.4(0.1-1.5)$ & & 0.224 & & $1.3(0.5-3.2)$ & & 0.445 \\
Obese & $4.5(1.3-15.2)$ & & 0.015 & & $1.2(0.3-5.0)$ & & 0.758 \\
\hline
\end{tabular}

$\mathrm{BMI}$, body mass index; TPA, total physical activity; $J 2 \mathrm{~F}$, jumping a distance of $7 \mathrm{~m}$ on 2 feet; SRT, $10 \times 5 \mathrm{~m}$ shuttle run test; all analyses were adjusted for age, gender and TPA. Bold figures indicate significant $P$-values.

Table 3. Association between performance in SRT $+J 2 F$ and obesity status in preschoolers.

\begin{tabular}{llcc} 
& & & \\
BMI & ORT $(95 \% \mathrm{Cl})$ & $R_{\mathrm{N}}^{2}$ & $P$-value \\
\hline Normal weight & 1 & 0.27 & 1 \\
Overweight & $0.3(0,6-2.1)$ & & 0.277 \\
Obese & $6.4(1.3-36.6)$ & & 0.035 \\
\hline
\end{tabular}

$\mathrm{BMI}$, body mass index; SRT, shuttle run test; J2F, jumping a distance of $7 \mathrm{~m}$ on 2 feet; TPA, total physical activity; all analyses were adjusted for age, gender and TPA. Bold figures indicate significant $P$-values.

counterparts. No statistical significant association was found for J2F (Table 2). In addition, multinomial logistic regression analysis (Table 3) showed that obese preschoolers were 6 times more likely to be classified as having low MF level than their non-overweight counterparts even after adjustment for age, gender and TPA.

\section{Discussion}

This study aimed to examine the association between MF and obesity status among Portuguese preschool children. The most important finding was that obese preschoolers were 6 times more likely to be associated with poor MF compared to their normal weight peers even after a set of adjustments such as gender and TPA. This outcome is worth highlighting because secular trends for most of the basic motor abilities showed a decline over time (Klein et al., 2015). Therefore, poor MF coupled with excess body fat along with sedentary lifestyle are significant predictors of developing coronary heart disease (Katzmarzyk et al., 2001; Ortega et al., 2008). There is also evidence that low MF results in a higher risk of later obesity in growing pre-pubertal children (Johnson et al., 2000; Moliner-Urdiales et al., 2011). In addition, our study showed that the association individually analysed was stronger in SRT and not statistically significant in $\mathrm{J} 2 \mathrm{~F}$ test. Our data has shown that the current prevalence of overweight and obesity was $22.7 \%$ and $9.7 \%$, respectively.

Instead of evaluating only MF, the majority of the studies analysed physical fitness (either cardiorespiratory fitness (CRF) or muscular fitness (MusF)) through a set of tests using different methodologies. It has been well described that both CRF and MuscF are important markers of health in children and adolescents. A recent study showed that low aerobic fitness in late adolescence is associated with an increased risk of early death. Furthermore, the risk of early death was higher in fit obese individuals than in unfit normal-weight individuals (Högström, Nordström, \& Nordström, 2015). To date only a few studies have assessed those associations in preschool children. However, those studies that examined either the association between CRF and/or MuscF with body composition have shown that obese children had lower physical fitness (Agha-Alinejad et al., 2015; Krombholz, 2013; Niederer et al., 2012; Ortega et al., 2015; Reeves, Broeder, Kennedy-Honeycutt, East, \& Matney, 1999).

In our study, however, it is difficult to find data providing information on the association of MF and obesity among preschoolers having used on a single MF test. Rather, it focuses on compound activities that recruit various combinations of multiple factors, such as strength, endurance, motor coordination, balance and agility (Fjortoft et al., 2011). Hence, we used two MF tests: jumping distance, which is an important skill to assess a child's ability to co-ordinate the leg-arm movement aiming the assessment of their sense of rhythm and timing as well; the $10 \times 5 \mathrm{~m} \mathrm{SRT}$ which is usually used as a measure of speed and agility (Fjortoft et al., 2011). Our data has showed a significant association between lower performance in SRT $(\mathrm{OR}=4.5)$ while we have not found significant association between obesity and J2F (OR: 1.2). However, when the two tests are taken together, data has shown that obese children were 6 times more likely to be associated with low MF (Table 3). Our findings are in line with previous studies in preschoolers (Agha-Alinejad et al., 2015; Halme, Parkkisenniemi, Kujala, \& Nupponen, 2009). However, to the best of our knowledge, there is only one study analysing the individual items of MF in overweight and obese preschoolers (Bayer et al., 2009) A recent study in Spanish kindergarten children showed an association of health-related physical fitness components, mainly muscular strength, the $4 \times 10 \mathrm{~m}$ SRT and CRF (i.e. speed-agility) with total and central body fat (Martinez-Tellez et al., 2015). The observed association between speed-agility and (i.e. $4 \times 10 \mathrm{~m}$ SRT) total obesity status also agrees with data found in Thai preschoolers (Yamborisut, Sakamoto, Wimonpeerapattana, \& Tontisirin, 2010) and might express, as suggested elsewhere, that obese preschool children have a lower performance on the test requiring propulsive movement of body mass in adolescents (Moliner-Urdiales et al., 2011). In our study, we could verify that there were statistically significant differences between MF categories. Children with high MF spent more time in PA than children who presented low MF. This data is in line with some studies that have shown children with low MF who had had also low levels of PA (Williams et al., 2008) and consequently spent less time outdoors as has been shown in preschoolers (Cleland et al., 2008). Moreover, the acquisition of basic skills will promote a worse motor performance.

We can assume that the importance of MF should be recognised as a target for intervention focusing in preventing the development of obesity in children. Furthermore, motor skill acquisition and PA play key roles in child development and promotion of health (Roth et al., 2015).

In addition, MF components are associated with different health findings. PA programs should be designed to improve not only the levels of cardiorespiratory and MusF but also be focused on MF, at least on these age groups (Ortega et al., 2008). Hence, the researchers in these age groups have focused on the compliance of PA guidelines and have not given so much attention to basic daily motor capacities. This panorama should be changed, once these capacities are also associated with diverse health indicators (Klein et al., 2015). Children with low MF have a tendency to be less physically active, to have increased obesity status 
and reduced aerobic fitness, which are all associated with cardiovascular risk factors. These factors are critical for leading to a vicious circuit that must be targeted in an earlier intervention (Pietilainen et al., 2008). If MF is seen as a PA compliance guideline, the preschoolers will be more likely to be physically active and healthy. The foundation for the basic movement skills is created during this period and should be specialised in later motor development. It is of high importance that children possess the best possible motor abilities and skills by the time they reach school age, since during the school period this development slows down, and further progress is greatly dependent on motor-ability-skill-base previously formed in preschool (Hinkley, Crawford, Salmon, Okely, \& Hesketh, 2008). In the same way, a recent study has shown that obesity preceded the decline in motor skills rather than the reverse and suggested that early childhood obesity intervention efforts might help prevent decay in motor proficiency that, in turn, may positively impact children's PA and overall fitness levels (Cheng et al., 2016). Thus, these data lead to the implementation of the motor repertoire as timely as possible in these ages, once it has an enormous potential and should be recognised as a fundamental target on PA programs within school. This component connected with intervention in this area should entail crucial advantages to both a healthy development and health across childhood and to a participant's health and quality of life (Robinson et al., 2015).

The strengths of this study focus on the assessment of MF in preschool children. Nevertheless, some limitations should be recognised. The study includes preschool children from only one metropolitan area, which makes it difficult to generalise the findings to other samples. The use of only two MF tests might be a limitation and other MF tests might be more sensitive than the two used in this study. Furthermore, the study was cross-sectional and it was not possible to infer causal relationships using such a model design.

\section{Conclusions}

Our results suggest that obesity among preschoolers was associated with lower MF. Obese children were 6 times more likely to be classified as having lower performance in the combined MF variable, since MF consists of physical fitness components that have a relationship with motor skill improvement. Therefore, our findings provide evidence to support the establishment of a tailored MF test in epidemiological and intervention studies in preschool children, as it seems that they are associated to obesity status since early ages. Nonetheless, longitudinal studies are needed to confirm these findings.

\section{Acknowledgements}

The group thanks the children, their parents as well as the staff from all kindergartens. This study was supported by Centre in Physical Activity, Health and Leisure (Faculty of Sport, University of Porto) and by the following Portuguese Foundation for Science and Technology (FCT) grants: UID/DTP/00617/2013; SFRH/BD/86538/2012 and Amanda Santos thanks the Brazilian Government Scholarship Program Ciência sem Fronteiras (CAPES) No. 6099/13-0.

\section{Disclosure statement}

No potential conflict of interest was reported by the authors.

\section{Funding}

This work was supported by the Centre in Physical Activity, Health and Leisure under Grant [UID/DTP/00617/2013]; Foundation for Science and Technology under Grant [SFRH/BD/86538/2012] and Coordination for the Improvement of Higher Education Personnel under Grant [number 6099/ 13-0].

\section{ORCID}

Jorge Mota (D) http://orcid.org/0000-0001-7571-9181

\section{References}

Agha-Alinejad, H., Farzad, B., Salari, M., Kamjoo, S., Harbaugh, B. L., \& Peeri, M. (2015). Prevalence of overweight and obesity among Iranian preschoolers: Interrelationship with physical fitness. Journal of Research in Medical Sciences, 20(4), 334-341.

Bayer, O., Bolte, G., Morlock, G., Rückinger, S., \& Kries, R. V. (2009). A simple assessment of physical activity is associated with obesity and motor fitness in pre-school children. Public Health Nutrition, 12(8), 1242-1247. doi:10.1017/S1368980008003753

Byun, W., Beets, M. W., \& Pate, R. R. (2015). Sedentary behavior in preschoolers: How many days of accelerometer monitoring is needed? International Journal of Environmental Research and Public Health, 12 (10), 13148-13161. doi:10.3390/ijerph121013148

Byun, W., Dowda, M., \& Pate, R. R. (2011). Correlates of objectively measured sedentary behavior in US preschool children. Pediatrics, 128(5), 937-945. doi:10.1542/peds.2011-0748

Cadenas-Sánchez, C., Artero, E. G., Concha, F., Leyton, B., \& Kain, J. (2015). Anthropometric characteristics and physical fitness level in relation to body weight status in chilean preschool children. Nutrición Hospitalaria, 32(n01), 346-353.

Caspersen, C. J., Powell, K. E., \& Christenson, G. M. (1985). Physical activity, exercise, and physical fitness: Definitions and distinctions for healthrelated research. Public Health Reports, 100(2), 126-131.

Cheng, J., East, P., Blanco, E., Sim, E. K., Castillo, M., Lozoff, B., \& Gahagan, S. (2016). Obesity leads to declines in motor skills across childhood. Child: Care, Health and Development, 42(3), 343-350.

Cleland, V., Crawford, D., Baur, L. A., Hume, C., Timperio, A., \& Salmon, J. (2008). A prospective examination of children's time spent outdoors, objectively measured physical activity and overweight. International Journal of Obesity, 32(11), 1685-1693. doi:10.1038/ijo.2008.171

Cole, T. J., Bellizzi, M. C., Flegal, K. M., \& Dietz, W. H. (2000). Establishing a standard definition for child overweight and obesity worldwide: International survey. British Medical Association, 320(7244), 1240-1243.

Fjørtoft, I., Pedersen, A. V., Sigmundsson, H., \& Vereijken, B. (2011). Measuring physical fitness in children who are 5 to 12 years old with a test battery that is functional and easy to administer. Journal of the American Physical Therapy Association, 91(7), 1087-1095.

Fjørtoft, l., Pedersen, A. V., Sigmundsson, H., \& Vereijken, B. (2003). Testing children's physical fitness: developing a new test for 4-12 year old children (pp. 1256). Oslo, Norway:: The Norwegian Social and Health Ministry.

Haga, M. (2008). The relationship between physical fitness and motor competence in children. Child: Care Health and Development, 34(3), 329-334.

Haga, M. (2009). Physical fitness in children with high motor competence is different from that in children with low motor competence. Journal of the American Physical Therapy Association, 89(10), 1089-1097.

Halme, T., Parkkisenniemi, S., Kujala, U. M., \& Nupponen, H. (2009). Relationships between standing broad jump, shuttle run and body mass index in children aged three to ei. The Journal of Sports Medicine and Physical Fitness, 49(4), 395-400.

Hinkley, T., Crawford, D., Salmon, J., Okely A. D., \& Hesketh, K. (2008). Preschool children and physical activity: A review of correlates. 
American Journal of Preventive Medicine, 34(5), 435-441. doi:10.1016/j. amepre.2008.02.001

Högström, G., Nordström, A., \& Nordström, P. (2015). Aerobic fitness in late adolescence and the risk of early death: A prospective cohort study of 1.3 million Swedish men. International Journal of Epidemiology, 4-7.

Janz, K. F. (1994). Validation of the CSA accelerometer for assessing children's physical activity. Medicine and Science in Sports Exercise, 26 (3), 369-375. doi:10.1249/00005768-199403000-00015

Johnson, M. S., Figueroa-Colon, R., Herd S. L., Fields D. A., Sun, M., Hunter, G. R., \& Goran M., I. (2000). Aerobic fitness, not energy expenditure, influences subsequent increase in adiposity in black and white children. Pediatrics, 106(4), E50. doi:10.1542/peds.106.4.e50

Katzmarzyk, P. T., Gagnon, J., Leon, A. S., Skinner, J. S., Wilmore, J. H., Rao, D. C., \& Bouchard, C. (2001). Fitness, fatness, and estimated coronary heart disease risk: The HERITAGE family study. Medicine \& Science in Sports \& Exercise, 33(4), 585-590. doi:10.1097/00005768-200104000-00012

Klein, D., Manz, K., Ferrari, N., Strüder, H., \& Graf, C. (2015). Effects of health promotion projects in preschools on body mass index and motor abilities. The Journal of Sports Medicine and Physical Fitness, 55(1-2), 103-112.

Kriemler, S., Manser-Wenger, S., Zahner, L., Braun-Fahrländer, C., Schindler, C., \& Puder J. J. (2008). Reduced cardiorespiratory fitness, low physical activity and an urban environment are independently associated with increased cardiovascular risk in children. Diabetologia, 51(8), 14081415. doi:10.1007/s00125-008-1067-z

Krombholz, H. (2013). Motor and cognitive performance of overweight preschool children. Perceptual \& Motor Skills, 116(1), 40-57. doi:10.2466/ 22.25.PMS.116.1.40-57

Martinez-Tellez, B., Sanchez-Delgado, G., Cadenas-Sanchez, C., MoraGonzalez, J., Martín-Matillas, M., Löf, M., ... Ruiz, J. R. (2015). Healthrelated physical fitness is associated with total and central body fat in preschool children aged 3 to 5 years. Pediatric Obesity, 3-6.

Moliner-Urdiales, D., Ruiz, J. R., Vicente-Rodriguez, G., Ortega, F. B., ReyLopez, J. P., España-Romero, V., ... HELENA Study Group. (2011). Associations of muscular and cardiorespiratory fitness with total and central body fat in adolescents: The HELENA study. British Journal of Sports Medicine, 45(2), 101-108. doi:10.1136/bjsm.2009.062430

Niederer, I., Kriemler, S., Zahner, L., Burgi, F., Ebenegger, V., Marques, P., \& Puder, J. J. (2012). BMl group-related differences in physical fitness and physical activity in preschool-age children: A cross-sectional analysis. Research Quarterly for Exercise and Sport, 83(1), 12-19. doi:10.1080/ 02701367.2012.10599820

O'Connor, T. M., Cerin, E., Hughes, S. O., Robles, J., Thompson, D. I., Mendoza, J. A., ... Lee, R. E. (2014). Psychometrics of the preschooler physical activity parenting practices instrument among a Latino sample. The International Journal of Behavioral Nutrition and Physical Activity, 11, 3. doi:10.1186/14795868-11-3

Ortega, F. B., Cadenas-Sanchez, C., Sanchez-Delgado, G., Mora-Gonzalez, J., Martinez-Tellez, B., Artero, E. G., ... Ruiz, J. R. (2015). Systematic review and proposal of a field-based physical fitness-test battery in preschool children: The PREFIT battery. Sports Medicine, 45(4), 533-555. doi:10.1007/s40279-014-0281-8

Ortega, F. B., Ruiz, J. R., \& Castillo, M. J. (2013). [Physical activity, physical fitness, and overweight in children and adolescents: Evidence from epidemiologic studies]. Endocrinologia Y Nutricion, 60(8), 458-469. doi:10.1016/j.endonu.2012.10.006

Ortega, F. B., Ruiz, J. R., Castillo, M. J., \& Sjöström, M. (2008). Physical fitness in childhood and adolescence: A powerful marker of health. International Journal of Obesity, 32(1), 1-11. doi:10.1038/sj. ijo.0803774

Ortega, F. B., Tresaco, B., Ruiz, J. R., Moreno, L. A., Martin-Matillas, M., Mesa, J. L., ... AVENA Study Group. (2007). Cardiorespiratory fitness and sedentary activities are associated with adiposity in adolescents. Obesity, 15(6), 15891599. doi:10.1038/oby.2007.188

Pate, R. R., O'Neill, J. R., Brown, W. H., Pfeiffer, K. A., Dowda, M., \& Addy, C. L. (2015). Prevalence of compliance with a new physical activity guideline for preschool-age children. Childhood Obesity, 11(4), 415-420. doi:10.1089/chi.2014.0143

Pietilainen, K. H., Kaprio, J., Borg, P., Plasqui, G., Yki-Jarvinen, H., Kujala, U. M., ... Rissanen, A. (2008). Physical inactivity and obesity: A vicious circle. Obesity, 16(2), 409-414. doi:10.1038/oby.2007.72

Purslow, L. R., Hill, C., Saxton, J., Corder, K., \& Wardle, J. (2008). Differences in physical activity and sedentary time in relation to weight in 8-9 year old children. International Journal of Behavioral Nutrition and Physical Activity, 5, 67. doi:10.1186/1479-5868-5-67

Reeves, L., Broeder, C. E., Kennedy-Honeycutt, L., East, C., \& Matney, L. (1999). Relationship of fitness and gross motor skills for five- to six-yr.old children. Perceptual and Motor Skills, 89(3), 739-747. doi:10.2466/ pms.1999.89.3.739

Robinson, L. E., Stodden, D. F., Barnett, L. M., Lopes, V. P., Logan, S. W., Rodrigues, L. P., \& D'Hondt, E. (2015). Motor competence and its effect on positive developmental trajectories of health. Sports Medicine, 45(9), 12731284. doi:10.1007/s40279-015-0351-6

Roth, K., Kriemler, S., Lehmacher, W., Ruf, K. C., Graf, C., \& Hebestreit, H. (2015). Effects of a physical activity intervention in preschool children. Medicine \& Science in Sports \& Exercise, 47(12), 2542-2551. doi:10.1249/ MSS.0000000000000703

Sardinha, L. B., Baptista, F., \& Ekelund, U. (2008). Objectively measured physical activity and bone strength in 9-year-old boys and girls. Pediatrics, 122(3), e728-e736. doi:10.1542/peds.2007-2573

Smith, J. J., Eather, N., Morgan, P. J., Plotnikoff, R. C., Faigenbaum, A, D., \& Lubans, D. R. (2014). The health benefits of muscular fitness for children and adolescents: A systematic review and meta-analysis. Sports Medicine, 44(9), 1209-1223. doi:10.1007/s40279-014-0196-4

Vale, S., Santos, R., Silva, P., Soares-Miranda, L., \& Mota, J. (2009). Preschool children physical activity measurement: Importance of epoch length choice. Pediatric Exercise Science, 21(4), 413-420. doi:10.1123/ pes.21.4.413

Williams, H.G., Pfeiffer, K. A., O'Neill, J. R., Dowda, M., Mclver, k. L., Brown, W. H., \& Pate, R. R. (2008). Motor skill performance and physical activity in preschool children. Obesity (Silver Spring), 16(6), 1421-1426. doi:10.1038/oby.2008.214

Yamborisut, U., Sakamoto, N., Wimonpeerapattana, W., \& Tontisirin, k. (2010). Waist circumference and body fat distribution indexes as screening tools for the overweight and obesity in Thai preschool children. Obesity Research \& Clinical Practice, 4(4), e307-e314. doi:10.1016/j.orcp.2010.08.001 